\title{
On the numerical ranges of matrices in max algebra
}

\author{
D. Thaghizadeh ${ }^{1} \cdot$ M. Zahraei ${ }^{1}$ - A. Peperko Zn $^{2,3}$ - N. Haj Aboutalebi ${ }^{4}$
}

Received: 6 January 2020 / Accepted: 30 June 2020 / Published online: 18 July 2020

(c) The Author(s) 2020

\begin{abstract}
Let $M_{n}\left(\mathbb{R}_{+}\right)$be the set of all $n \times n$ nonnegative matrices. Recently, in Tavakolipour and Shakeri (Linear Multilinear Algebra 67, 2019, https://doi.org/10.1080/030 81087.2018.1478946), the concept of the numerical range in tropical algebra was introduced and an explicit formula describing it was obtained. We study the isomorphic notion of the numerical range of nonnegative matrices in max algebra and give a short proof of the known formula. Moreover, we study several generalizations of the numerical range in max algebra. Let $1 \leq k \leq n$ be a positive integer and $C \in M_{n}\left(\mathbb{R}_{+}\right)$. We introduce the notions of max $k$-numerical range and max $C-$ numerical range. Some algebraic and geometric properties of them are investigated. Also, max numerical range $W_{\max }(\Sigma)$ of a bounded set $\Sigma$ of $n \times n$ nonnegative matrices is introduced and some of its properties are also investigated.
\end{abstract}

Keywords Numerical range $\cdot$ Tropical algebra $\cdot$ Max algebra $\cdot k$-numerical range . C-numerical range

Mathematics Subject Classification $15 \mathrm{~A} 60 \cdot 15 \mathrm{~A} 18 \cdot 15 \mathrm{~A} 80$

Communicated by Masatoshi Fujii.

M. Zahraei

mzahraei326@gmail.com

D. Thaghizadeh

t.davood1411@gmail.com

A. Peperko

aljosa.peperko@fs.uni-lj.si; aljosa.peperko@fmf.uni-lj.si

N. Haj Aboutalebi

aboutalebi.n@yahoo.com

1 Department of Mathematics, Ahvaz Branch, Islamic Azad University, Ahvaz, Iran

2 Faculty of Mechanical Engineering, University of Ljubljana, Aškerčeva 6, 1000 Ljubljana, Slovenia

3 Institute of Mathematics, Physics and Mechanics Jadranska 19, 1000 Ljubljana, Slovenia

4 Department of Mathematics, Shahrood Branch, Islamic Azad University, Shahrood, Iran 


\section{Introduction and preliminaries}

The algebraic system max algebra and its isomorphic versions (max plus algebra, tropical algebra) provide an attractive way of describing a class of nonlinear problems appearing for instance in manufacturing and transportation scheduling, information technology, discrete event dynamic systems, combinatorial optimization, mathematical physics, DNA analysis, ...(see e.g. $[1,3,5,6,10,11]$ and the references cited there). It has been used to describe these conventionally nonlinear problems in a linear fashion.

Max algebra consists of the set of nonnegative real numbers equipped with the basic operations of multiplications $a \otimes b=a b$, and maximization $a \oplus b=\max \{a, b\}$. For $A=\left(a_{i j}\right) \in M_{m \times n}(\mathbb{R})$, we say that $A$ is positive (nonnegative) and write $A>0(A \geq 0)$ if $a_{i j}>0\left(a_{i j} \geq 0\right)$ for $1 \leq i \leq m, 1 \leq j \leq n$. Let $\mathbb{R}_{+}$be the set of all nonnegative real numbers and $M_{m \times n}\left(\mathbb{R}_{+}\right)$be the set of all $m \times n$ nonnegative real matrices. The notions $M_{n}\left(\mathbb{R}_{+}\right)$and $\mathbb{R}_{+}^{n}$ are considered for $M_{n \times n}\left(\mathbb{R}_{+}\right)$ and $M_{n \times 1}\left(\mathbb{R}_{+}\right)$, respectively. Let $A=\left(a_{i j}\right) \in M_{m \times n}\left(\mathbb{R}_{+}\right)$and $B=\left(b_{i j}\right) \in M_{n \times l}\left(\mathbb{R}_{+}\right)$. The product of $A$ and $B$ in max algebra is denoted by $A \otimes B$, where $(A \otimes B)_{i j}=\max _{k=1, \ldots, n} a_{i k} b_{k j}$. Similarly the vector $A \otimes x$ is defined by $(A \otimes x)_{i}=\max _{k=1, \ldots, n} a_{i k} x_{k}$ for $i=1, \ldots, m$ if $x \in \mathbb{R}_{+}^{n}$. If $A, B \in M_{n}\left(\mathbb{R}_{+}\right)$then the max sum $A \oplus B$ in max algebra is defined by $(A \oplus B)_{i j}=\max \left\{a_{i j}, b_{i j}\right\}$ for $i, j=1, \ldots, n$. The notation $A_{\otimes}^{2}$ means $A \otimes A$, and $A_{\otimes}^{k}$ denotes the $k$ th power of $A$ in max algebra. For $A \in M_{n}\left(\mathbb{R}_{+}\right)$and $x \in \mathbb{R}_{+}^{n}$, let us denote $\|A\|=\max _{i, j=1, \ldots, n} a_{i j}$ and $\|x\|=\max _{i=1, \ldots, n} x_{i}$. Let $r_{x}(A)$ denote the local spectral radius of $A$, i.e., $r_{x}(A)=\limsup _{j \rightarrow \infty}\left\|A_{\otimes}^{j} \otimes x\right\|^{i=1, j}$. It was shown in [11] for $x=\left[x_{1}, \ldots, x_{n}\right]^{t} \in \mathbb{R}_{+}^{n}, x \neq 0$ that $r_{x}(A)=\lim _{j \rightarrow \infty}\left\|A_{\otimes}^{j} \otimes x\right\|^{1 / j}$ and that $r_{x}(A)=\max \left\{r_{e_{i}}(A): i=1, \ldots, n, x_{i} \neq 0\right\}$, where $e_{i}$ denotes the $i$ th standard basis vector and $x_{i}$ denotes the $i$ th coordinate of $x$. We say that $\mu \geq 0$ is a geometric max eigenvalue of $A$ if $A \otimes x=\mu x$ for some $x \neq 0, x \geq 0$. Let $\sigma_{\max }(A)$ denote the set of geometric max eigenvalues of $A$. The following result of Gunawardena was restated and reproved in [11, Theorem 2.7].

Theorem 1 If $A \in M_{n}\left(\mathbb{R}_{+}\right)$, then

$$
\sigma_{\max }(A)=\left\{\mu: \text { there exists } j \in\{1, \ldots, n\}, \mu=r_{e_{j}}(A)\right\} .
$$

We define the standard vector multiplicity of geometric max eigenvalue $\mu$ as the number of indices $j$ such that $\mu=r_{e_{j}}(A)$.

The role of the spectral radius of $A$ in max algebra is played by the maximum cycle geometric mean $\mu(A)$, which is defined by

$$
\mu(A)=\max \left\{\left(a_{i_{1} i_{k}} \cdots a_{i_{3} i_{2}} a_{i_{2} i_{1}}\right)^{1 / k}: k \in \mathbb{N} \text { and } i_{1}, \ldots, i_{k} \in\{1, \ldots, n\}\right\}
$$

and is equal to 


$$
\mu(A)=\max \left\{\left(a_{i_{1} i_{k}} \cdots a_{i_{3} i_{2}} a_{i_{2} i_{1}}\right)^{1 / k}: k \leq n \text { and } i_{1}, \ldots, i_{k} \in\{1, \ldots, n\} \text { mutually distinct }\right\} .
$$

A digraph $\mathcal{G}(A)=(N(A), E(A))$ associated to $A$ is defined by setting $N(A)=\{1, \ldots, n\}$ and letting $(i, j) \in E(A)$ whenever $a_{i j}>0$. When this digraph contains at least one cycle, one distinguishes critical cycles, where the maximum in (1) is attained. A graph with just one node and no edges will be called trivial. A bit unusually, but in consistency with [3] and [11], a matrix $A \in M_{n}\left(\mathbb{R}_{+}\right)$is called irreducible if $\mathcal{G}(A)$ is trivial ( $A$ is $1 \times 1$ zero matrix) or strongly connected (for each $i, j \in N(A), i \neq j$, there is a path in $\mathcal{G}(A)$ that starts in $i$ and ends in $j)$.

It is known that $\mu(A)$ is the largest geometric max eigenvalue of $A$, i.e., $\mu(A)=\max \left\{\mu: \mu \in \sigma_{\max }(A)\right\}$ and so we have $\mu(A)=\max _{j=1, \ldots, n} r_{e_{j}}(A)$. Moreover, if $A$ is irreducible, then $\mu(A)$ is the unique max eigenvalue and every max eigenvector is positive (see e.g. [3]).

The max permanent of $A$ is

$$
\operatorname{perm}(A)=\max _{\sigma \in S_{n}} a_{1 \sigma(1)} \cdots a_{n \sigma(n)},
$$

where $S_{n}$ is the group of permutations on $\{1, \ldots, n\}$. The characteristic maxpolynomial of $A$ (see e.g. [3, 14, 15]) is a max polynomial

$$
\chi_{A}(x)=\operatorname{perm}(x I \oplus A),
$$

where $I$ denotes the identity matrix. We call its tropical roots (the points of nondifferentiability of $\chi_{A}(x)$ considered as a function on $[0, \infty)$ ) algebraic max eigenvalues (or also tropical eigenvalues) of $A$. The set of all algebraic max eigenvalues is denoted by $\sigma_{\text {trop }}(A)$. For $\lambda \in \sigma_{\text {trop }}(A)$ its multiplicity as a tropical root of $\chi_{A}(x)$ (see e.g [3, 14, $15])$ is called an algebraic multiplicity of $\lambda$. It is known that $\sigma_{\max }(A) \subset \sigma_{\text {trop }}(A)[15$, Remark 2.3] and that $\mu(A)=\max \left\{\lambda: \lambda \in \sigma_{\text {trop }}(A)\right\}$, but in general, the sets $\sigma_{\text {max }}(A)$ and $\sigma_{\text {trop }}(A)$ may differ.

Let $A \in M_{n}\left(\mathbb{R}_{+}\right)$. The max-numerical range $W_{\text {max }}(A)$ of $A$ was defined in [15] (actually its isomorphic version in the setting of max-plus (tropical) algebra) and it was shown there that $\sigma_{\text {trop }}(A) \subset W_{\text {max }}(A)[15$, Theorem 3.10]. It was proved in [15, Theorem 3.7] that given $A \in M_{n}\left(\mathbb{R}_{+}\right)$

$$
W_{\max }(A)=\left[\min _{i \in\{1, \ldots, n\}} a_{i i}, \max _{i, j \in\{1, \ldots, n\}} a_{i j}\right] .
$$

In the current article we provide a short proof of this fact. This proof provides also new insights, which enables us to consider several generalizations of the maxnumerical range and to provide interesting results for these generalizations.

As it will be evident from below the article is partly expository and is organized as follows. In Sect. 2 we give a short proof of the formula (2) (Theorem 2) and obtain some interesting results. In the third section we recall the definition of the joint numerical range of a $k$-tuple $\left(A_{1}, \ldots, A_{k}\right)$, where $A_{i} \in M_{n}, i=1, \ldots, k$, and we apply Theorem 2 to obtain a new formula for max joint numerical range $W_{\max }(\Sigma)$ of a bounded set $\Sigma$ of $n \times n$ nonnegative matrices (11). We move on in Section 4 
to introduce some definitions and facts, which we need in our proofs and study the max $k$-numerical range $W_{\max }^{k}(A)$, where $k \leq n$ is a positive integer. We explicitly describe a formula for $W_{\max }^{k}(A)$ (Theorem 3) and then use this to state some of its basic properties (Theorem 4). Related interesting results are also obtained for the max $k$-geometric spectrum and $k$-tropical spectrum of $A \in M_{n}\left(\mathbb{R}_{+}\right)$. In the last section we introduce and study the max $c$-numerical range and max $C$-numerical range of nonnegative matrices, where $c \in \mathbb{R}_{+}^{n}$ and $C \in M_{n}\left(\mathbb{R}_{+}\right)$. Also, we investigate some basic algebraic and geometrical properties of these sets.

\section{Max-numerical range}

Let $M_{n}(\mathbb{C})$ be the vector space of all $n \times n$ complex matrices. The numerical range of a square matrix $A \in M_{n}(\mathbb{C})$ is defined by

$$
W(A)=\left\{x^{*} A x: x \in \mathbb{C}^{n}, x^{*} x=1\right\}
$$

It is known that $W(A)$ is compact, convex and contains the spectrum of $A$. In [15], the numerical range of a given square matrix was introduced and described in the setting of max-plus algebra. We study here its isomorphic version in max algebra setting and provide a short proof of one of their main results [15, Theorem 3.7] in Theorem 2.

Definition 1 Let $A \in M_{n}\left(\mathbb{R}_{+}\right)$be a non-negative matrix. The max numerical range $W_{\text {max }}(A)$ of $A$ is defined by

$$
W_{\max }(A)=\left\{x^{t} \otimes A \otimes x: x \in \mathbb{R}_{+}^{n}, x^{t} \otimes x=1\right\}
$$

It's obvious that

$$
\begin{aligned}
W_{\max }(A) & =\left\{x^{t} \otimes A \otimes x: x \in \mathbb{R}_{+}^{n}, x^{t} \otimes x=1\right\} \\
& =\left\{\frac{x^{t}}{\sqrt{x^{t} \otimes x}} \otimes A \otimes \frac{x}{\sqrt{x^{t} \otimes x}}: 0 \neq x \in \mathbb{R}_{+}^{n}\right\} \\
& =\left\{\frac{1}{x^{t} \otimes x} x^{t} \otimes A \otimes x: 0 \neq x \in \mathbb{R}_{+}^{n}\right\}
\end{aligned}
$$

Remark 1 (i) If $x \in \mathbb{R}_{+}^{n}$, then $x^{t} \otimes x=1$ means

$$
\max \left\{x_{1}, x_{2}, \ldots, x_{n}\right\}=1,
$$

i.e., for all $1 \leq i \leq n, 0 \leq x_{i} \leq 1$ and $x_{j}=1$ for some $1 \leq j \leq n$.

(ii) Suppose that $A \in M_{n}\left(\mathbb{R}_{+}\right)$and $f_{A}: S \longrightarrow \mathbb{R}_{+}$, where

$$
S=\left\{x \in \mathbb{R}_{+}^{n}, x^{t} \otimes x=1\right\}, f_{A}(x):=x^{t} \otimes A \otimes x .
$$


So $W_{\max }(A)$ is the image of the continuous function $f_{A}$. Since $S$ is a connected set, also $W_{\max }(A)$ is a connected set.

Next we provide a new short proof of (2) ([15, Theorem 3.7]).

Theorem 2 Let $A=\left(a_{i j}\right) \in M_{n}\left(\mathbb{R}_{+}\right)$be a nonnegative matrix. Then

$$
W_{\text {max }}(A)=[a, b] \subseteq \mathbb{R}_{+},
$$

where $a=\min _{1 \leq i \leq n} a_{i i}$ and $b=\max _{1 \leq i, j \leq n} a_{i j}$.

Proof By definition of $W_{\max }(A)$ we have

$$
W_{\max }(A)=\left\{\bigoplus_{i, j=1}^{n} a_{i j} x_{i} x_{j}: x_{i} \in \mathbb{R}_{+} \forall i=1,2, \ldots, n, \max \left\{x_{1}, x_{2}, \ldots, x_{n}\right\}=1\right\} .
$$

Let $z \in W_{\max }(A)$ be given. So,

$$
z=x^{t} \otimes A \otimes x=\bigoplus_{i, j=1}^{n} a_{i j} x_{i} x_{j},
$$

for some $x=\left[x_{1}, x_{2}, \ldots, x_{n}\right]^{t} \in \mathbb{R}_{+}^{n}$ with $\max \left\{x_{1}, x_{2}, \ldots, x_{n}\right\}=1$. It holds that $0 \leq x_{i} \leq 1$ for all $1 \leq i \leq n$ and $x_{i_{0}}=1$ for some $1 \leq i_{0} \leq n$. By taking

$$
z=\max \left\{a_{i j} x_{i} x_{j}: 1 \leq i, j \leq n, 0 \leq x_{i}, x_{j} \leq 1\right\}=a_{i_{1} j_{1}} x_{i_{1}} x_{j_{1}},
$$

for some $1 \leq i_{1}, j_{1} \leq n$, we have

$$
a \leq a_{i_{0} i_{0}}=a_{i_{0} i_{0}} x_{i_{0}} x_{i_{0}} \leq z=a_{i_{1} j_{1}} x_{i_{1}} x_{j_{1}} \leq a_{i_{1} j_{1}} \leq b,
$$

since $a=\min _{1 \leq i \leq n} a_{i i}$ and $b=\max _{1 \leq i, j \leq n} a_{i j}$. So $W_{\max }(A) \subseteq[a, b]$.

To prove the reverse inclusion, recall that the function $x \longmapsto f_{A}(x)=x^{t} \otimes A \otimes x$ is continuous on the compact connected set (Fig. 1)

$$
S=\left\{x \in \mathbb{R}_{+}^{n}, x^{t} \otimes x=1\right\} .
$$

Let $1 \leq k \leq n$ be such that $a=\min _{1 \leq i \leq n} a_{i i}=a_{k k}$. Then the vectors $x=[1,1, \ldots, 1]^{t}$ and $y=e_{k}=[0, \ldots, 0,1,0, \ldots, 0]^{t}$ satisfy $x, y \in S$ and

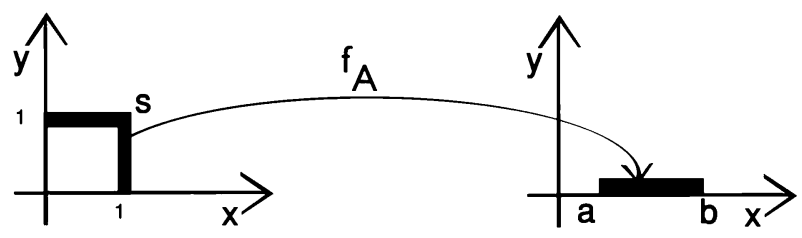

Fig. 1 The max numerical range of a $n \times n$ matrix $A, W_{\max }(A)$ 


$$
f_{A}(x)=x^{t} \otimes A \otimes x=\bigoplus_{i, j=1}^{n} a_{i j}=b
$$

and

$$
f_{A}(y)=y^{t} \otimes A \otimes y=a_{k k}=\min _{1 \leq i \leq n} a_{i i}=a .
$$

By Remark 1(ii), $W_{\max }(A)$ is a connected set and so $W_{\max }(A)=[a, b]$, which completes the proof.

Remark 2 Alternatively, in Theorem 2 , one can prove $[a, b] \subseteq W_{\max }(A)$, where

$$
a=\min _{1 \leq i \leq n} a_{i i}, b=\max _{1 \leq i, j \leq n} a_{i j}
$$

in the following constructive way.

Let $z \in[a, b]$ be given and let $a=\min _{1 \leq i \leq n} a_{i i}=a_{k k}$ and $b=\max _{1 \leq i, j \leq n} a_{i j}=a_{r s}$ for some $1 \leq k, r, s \leq n$. Now we consider four cases.

Case 1: If $r \neq s, r=k$ or $s=k$, by taking $\quad x=\left[\frac{z}{b}, \ldots, 1, \ldots, \frac{z}{b}\right]^{t}$, where $x_{k}=1, x_{i}=\frac{z}{b} \forall i \neq k$, we have

$$
x^{t} \otimes x=1, x^{t} \otimes A \otimes x=z .
$$

Case 2: If $r=s=k$, then by taking $x=[1, \ldots, 1]^{t}$ we have

$$
x^{t} \otimes x=1, x^{t} \otimes A \otimes x=z=a=b .
$$

Case 3: If $r=s, r \neq k$, then by letting $\alpha=\max \left\{a_{k r}, a_{r k}\right\}$ and taking

$$
x=\left\{\begin{array}{l}
{\left[0, \ldots, 1, \ldots, \sqrt{\frac{z}{b}}, \ldots, 0\right]^{t}, x_{k}=1, x_{r}=\sqrt{\frac{z}{b}}, x_{i}=0 \forall i \neq r, k z>\frac{\alpha^{2}}{b}} \\
{\left[0, \ldots, 1, \ldots, \frac{z}{\alpha}, \ldots, 0\right]^{t}, x_{k}=1, x_{r}=\frac{z}{\alpha}, x_{i}=0 \forall i \neq r, k \quad z \leq \frac{\alpha^{2}}{b},}
\end{array}\right.
$$

we have

$$
x^{t} \otimes x=1, x^{t} \otimes A \otimes x=z .
$$

Case 4: Finally, for $r \neq s, r \neq k, s \neq k$, by letting $\alpha=\max \left\{a_{k r}, a_{r k}, a_{r r}\right\}$ and by taking

$$
x= \begin{cases}{\left[0, \ldots, 1, \ldots, \frac{z}{\alpha}, \ldots, 0\right]^{t}, x_{k}=1, x_{r}=\frac{z}{\alpha}} & \alpha=a_{k r} \oplus a_{r k} \\ {\left[0, \ldots, 1, \ldots, \sqrt{\frac{z}{\alpha}}, \ldots, 0\right]^{t}, x_{k}=1, x_{r}=\sqrt{\frac{z}{\alpha}}} & \alpha=a_{r r}, z>\frac{\left(a_{k r} \oplus a_{r k}\right)^{2}}{\alpha} \\ {\left[0, \ldots, 1, \ldots, \frac{z}{a_{k r} \oplus a_{r k}}, \ldots, 0\right]^{t}, x_{k}=1, x_{r}=\frac{z}{a_{k r} \oplus a_{r k}}} & \alpha=a_{r r}, z \leq \frac{\left(a_{k r} \oplus a_{r k}\right)^{2}}{\alpha}\end{cases}
$$

if $a \leq z \leq \alpha$, and by taking 


$$
x=\left[0, \ldots, 1, \ldots, 1, \ldots, \frac{z}{b}, \ldots, 0\right]^{t}, x_{k}=x_{r}=1, x_{s}=\frac{z}{b}
$$

if $\alpha \leq z \leq b$, one can verify that

$$
x^{t} \otimes x=1, x^{t} \otimes A \otimes x=z,
$$

which completes the proof.

Corollary 1 Let $A=\operatorname{diag}\left(\lambda_{1}, \ldots, \lambda_{n}\right) \in M_{n}\left(\mathbb{R}_{+}\right)$be a diagonal matrix and $0 \leq \lambda_{1} \leq \cdots \leq \lambda_{n}$, then $W_{\max }(A)=\left[\lambda_{1}, \lambda_{n}\right]$.

Since the maximum of differentiable functions is locally Lipschitz continuous, the following proposition follows.

Proposition 1 Let $A=\left(a_{i j}\right) \in M_{n}\left(\mathbb{R}_{+}\right)$be a nonnegative matrix. The map $f_{A}: S \longrightarrow \mathbb{R}_{+}$where

$$
S=\left\{x \in \mathbb{R}_{+}^{n}, x^{t} \otimes x=1\right\}, f_{A}(x)=x^{t} \otimes A \otimes x
$$

is locally Lipschitz continuous on $S$.

In conventional algebra, a matrix $U \in M_{n}$ is called unitary if $U^{*} U=U U^{*}=I_{n}$. By analogy one can make the following definition in max algebra:

Definition 2 Let $U \in M_{n}\left(\mathbb{R}_{+}\right)$. If $U^{t} \otimes U=U \otimes U^{t}=I_{n}$, then $U$ is called unitary in max algebra and we denote

$$
\mathcal{U}_{n}=\left\{U \in M_{n}\left(\mathbb{R}_{+}\right): U^{t} \otimes U=U \otimes U^{t}=I_{n}\right\}
$$

The following result was established in [3].

Proposition 2 Let $A \in M_{n}\left(\mathbb{R}_{+}\right)$be a non-negative matrix. Then $A$ is unitary in max algebra if and only if $A$ is a permutation matrix.

The following proposition is an analogue of the property of unitary similarity invariance for the field of values, [8, Chapter1]. Its proof is straightforward and it is omitted.

Proposition 3 Let $A, P \in M_{n}\left(\mathbb{R}_{+}\right)$be nonnegative matrices and let $P$ be a permutation matrix. Then

$$
W_{\max }\left(P^{t} \otimes A \otimes P\right)=W_{\max }(A) .
$$

For $X, Y \subseteq \mathbb{R}_{+}$, recall that $X \oplus Y$ is defined as follows:

$$
X \oplus Y=\{x \oplus y: x \in X, y \in Y\} .
$$


In the following two results we collect some properties of $W_{\max }$. By Theorem 2, the proofs are straightforward and we omit them. Let us point out that (ii), (iv) and one inclusion in (i) from Proposition 4 have already been stated in [15].

Proposition 4 Let $A, B \in M_{n}\left(\mathbb{R}_{+}\right)$be nonnegative matrices and let $\alpha, \beta \in \mathbb{R}_{+}$. Then the following statements hold.

(i) $W_{\text {max }}(A \oplus B)=W_{\text {max }}(A) \oplus W_{\text {max }}(B)$.

(ii) If $\alpha \neq 0, \quad W_{\max }(\alpha A \oplus \beta I)=\alpha W_{\max }(A) \oplus\{\beta\}=\alpha W_{\max }\left(A \oplus \frac{\beta}{\alpha} I\right) . \quad$ Also, $W_{\max }(\beta I)=\{\beta\}$.

(iii) If $A=\left[\begin{array}{cc}D & 0_{n_{1} \times n_{2}} \\ 0_{n_{2} \times n_{1}} & C\end{array}\right]$, where $D \in M_{n_{1}}\left(\mathbb{R}_{+}\right), C \in M_{n_{2}}\left(\mathbb{R}_{+}\right), n=n_{1}+n_{2}$, then $W_{\max }(D) \oplus W_{\max }(C) \subseteq W_{\max }(A)$.

The equality holds, when $\min _{1 \leq i \leq n_{1}} d_{i i}=\min _{1 \leq i \leq n_{2}} c_{i i}$.

(iv) $\sigma_{\max }(A) \subseteq \sigma_{\text {trop }}(A) \subseteq W_{\text {max }}(A)$.

(v) $W_{\max }\left(A^{t}\right)=W_{\text {max }}(A)$.

(vi) If $\max _{1 \leq i, j \leq n} a_{i j}=a_{k k}$ for some $1 \leq k \leq n$, then $\max \left(W_{\max }\left(A_{\otimes}^{m}\right)\right)=a_{k k}^{m}$.

Proposition 5 Let $A, B \in M_{n}\left(\mathbb{R}_{+}\right)$be diagonal matrices. If $W_{\max }(A) \subseteq W_{\max }(B)$, then

$$
W_{\text {max }}\left(A_{\otimes}^{m}\right) \subseteq W_{\max }\left(B_{\otimes}^{m}\right), \quad \forall m \geq 1 .
$$

It turns out that in several cases the quotients $\frac{l\left(W_{\max }\left(A_{\otimes}^{m+1}\right)\right)}{l\left(W_{\max }\left(A_{\otimes}^{m}\right)\right)}$ and $\frac{l\left(W_{\max }\left(A_{\otimes}^{m+2}\right)\right)}{l\left(W_{\max }\left(A_{\otimes}^{m}\right)\right)}$, where $l(\cdot)$ denotes the length of an interval, have interesting asymptotic behaviour. We illustrate this in the following examples.

Example 1 Let $A=\left[\begin{array}{lll}4 & 2 & 5 \\ 3 & 7 & 1 \\ 9 & 6 & 8\end{array}\right]$. So $W_{\max }(A)=\left[4,3^{2}\right]$ and by computing $A_{\otimes}^{2}, A_{\otimes}^{3}, A_{\otimes}^{4}, \ldots, A_{\otimes}^{15}$, respectively, we have

$$
W_{\max }\left(A_{\otimes}^{2}\right)=\left[3^{2} \times 5,3^{2} \times 2^{3}\right], \quad W_{\max }\left(A_{\otimes}^{3}\right)=\left[7^{3}, 3^{2} \times 2^{6}\right], \quad W_{\max }\left(A_{\otimes}^{4}\right)=\left[7^{4}, 3^{2} \times 2^{9}\right], \ldots,
$$

and

$$
W_{\max }\left(A_{\otimes}^{15}\right)=\left[11.25 \times 8^{13}, 72 \times 8^{13}\right] .
$$

By a straightforward induction, we can show that

$$
A_{\otimes}^{m}=\left[\begin{array}{ccc}
45 \times 8^{m-2} & 30 \times 8^{m-2} & 40 \times 8^{m-2} \\
1080 \times 8^{m-4} & 11.25 \times 8^{m-2} & 15 \times 8^{m-2} \\
72 \times 8^{m-2} & 48 \times 8^{m-2} & 64 \times 8^{m-2}
\end{array}\right] \forall m \in \mathbb{N}, m \geq 15
$$

and so it follows that 


$$
W_{\text {max }}\left(A_{\otimes}^{m}\right)=\left[11.25 \times 8^{m-2}, 72 \times 8^{m-2}\right] \forall m \in \mathbb{N}, m \geq 15 .
$$

It follows from (5) that

$$
\lim _{m \rightarrow \infty} \frac{l\left(W_{\max }\left(A_{\otimes}^{m+1}\right)\right)}{l\left(W_{\max }\left(A_{\otimes}^{m}\right)\right)}=8 .
$$

Example 2 Let $A=\left[\begin{array}{llll}3 & 4 & 2 & 9 \\ 6 & 2 & 7 & 3 \\ 9 & 3 & 4 & 5 \\ 8 & 2 & 5 & 6\end{array}\right]$. By induction, we establish that

$$
A_{\otimes}^{2 m}=72^{m-2} A_{\otimes}^{4}, A_{\otimes}^{2 m+1}=72^{m-2} A_{\otimes}^{5}, \forall m \in \mathbb{N}, m \geq 3,
$$

where

$$
A_{\otimes}^{4}=\left[\begin{array}{llll}
5184 & 1728 & 3240 & 3888 \\
4536 & 1728 & 2835 & 3888 \\
3888 & 2592 & 2430 & 5832 \\
3456 & 2304 & 2160 & 5184
\end{array}\right], A_{\otimes}^{5}=\left[\begin{array}{llll}
31104 & 20736 & 19440 & 46656 \\
31104 & 18144 & 19440 & 40824 \\
46656 & 15552 & 29160 & 34992 \\
41472 & 13824 & 25920 & 31104
\end{array}\right] .
$$

A straightforward computation shows that (6) holds for $m=3$. Assume now that (6) holds for $m=3, \ldots, k$. Thus, by the inductive assumption, we obtain

$$
\begin{aligned}
A_{\otimes}^{2(k+1)} & =A_{\otimes}^{2 k} \otimes A_{\otimes}^{2}=72^{k-2} A_{\otimes}^{4} \otimes A_{\otimes}^{2} \\
& =72^{k-2} A_{\otimes}^{6}=72^{k-2} \times 72 A_{\otimes}^{4} \\
& =72^{k-1} A_{\otimes}^{4},
\end{aligned}
$$

and similarly

$$
A_{\otimes}^{2(k+1)+1}=72^{k-1} A_{\otimes}^{5}
$$

Using (6) we have

$$
W_{\max }\left(A_{\otimes}^{2 m}\right)=\left[72^{m-2} \times 1728,72^{m-2} \times 5832\right]
$$

and

$$
W_{\max }\left(A_{\otimes}^{2 m+1}\right)=\left[72^{m-2} \times 18144,72^{m-2} \times 46656\right] .
$$

It follows

$$
\lim _{m \rightarrow \infty} \frac{l\left(W_{\max }\left(A_{\otimes}^{2 m+1}\right)\right)}{l\left(W_{\max }\left(A_{\otimes}^{2 m}\right)\right)}=\frac{132}{19}, \quad \lim _{m \rightarrow \infty} \frac{l\left(W_{\max }\left(A_{\otimes}^{2 m+2}\right)\right)}{l\left(W_{\max }\left(A_{\otimes}^{2 m+1}\right)\right)}=\frac{114}{11}
$$

and 


$$
\lim _{m \rightarrow \infty} \frac{l\left(W_{\max }\left(A_{\otimes}^{2 m+2}\right)\right)}{l\left(W_{\max }\left(A_{\otimes}^{2 m}\right)\right)}=\lim _{m \rightarrow \infty} \frac{l\left(W_{\max }\left(A_{\otimes}^{2 m+3}\right)\right)}{l\left(W_{\max }\left(A_{\otimes}^{2 m+1}\right)\right)}=72 .
$$

So the limit $\lim _{m \rightarrow \infty} \frac{l\left(W_{\max }\left(A_{\otimes}^{m+1}\right)\right)}{l\left(W_{\max }\left(A_{\otimes}^{m}\right)\right)}$ does not exist, but the limit

$$
\lim _{m \rightarrow \infty} \frac{l\left(W_{\max }\left(A_{\otimes}^{m+2}\right)\right)}{l\left(W_{\max }\left(A_{\otimes}^{m}\right)\right)}
$$

exists and it is equal to 72 .

The Cyclicity theorem in max-algebra ([3, Theorem 8.3.5]) states the following: if $A \in M_{n}\left(\mathbb{R}_{+}\right)$is an irreducible matrix, then there exists $p \in \mathbb{N}$ and there exists $T \in \mathbb{N}$ such that

$$
A_{\otimes}^{m+p}=\mu(A)^{p} A_{\otimes}^{m}
$$

holds for every $m \geq T$. A matrix $A$ for which there exist $p$ and $T$ such that (7) holds for all $m \geq T$ is called ultimately periodic. Thus every irreducible matrix is ultimately periodic. The smallest $p$ such that (7) holds for all $m \geq T$ and some $T$ is called a period of $A$. It is known that a period of an irreducible matrix $A$ equals the cyclicity of $A$ (see[3, Chapter 8]).

More generally, the General cyclicity theorem ([3, Theorem 8.6.9]) states that $A \in M_{n}\left(\mathbb{R}_{+}\right)$is ultimately periodic if and only if each irreducible diagonal block of the Frobenius normal form of $A$ has the same geometric max eigenvalue (equal to $\mu(A))$. For definitions, we refer to[3].

Consequently, if $A \in M_{n}\left(\mathbb{R}_{+}\right)$is irreducible, then there exist natural numbers $p$ and $T$ such that $l\left(W_{\max }\left(A_{\otimes}^{m+p}\right)\right)=\mu(A)^{p} l\left(W_{\max }\left(A_{\otimes}^{m}\right)\right)$ for all $m \geq T$. Therefore, if $l\left(W_{\max }\left(A_{\otimes}^{m}\right)\right)>0$ for all $m \geq T$, then

$$
\lim _{m \rightarrow \infty} \frac{l\left(W_{\max }\left(A_{\otimes}^{m+p}\right)\right)}{l\left(W_{\max }\left(A_{\otimes}^{m}\right)\right)}=\mu(A)^{p} .
$$

In the following, we consider two special cases when (8) holds for $p=1$.

Proposition 6 Let $A=\left(a_{i j}\right) \in M_{n}\left(\mathbb{R}_{+}\right)$be a nonnegative matrix such that $l\left(W_{\max }(A)\right)>0$.

(i) If A is an upper triangular matrix or a lower triangular matrix, then

$$
\lim _{m \rightarrow \infty} \frac{l\left(W_{\max }\left(A_{\otimes}^{m+1}\right)\right)}{l\left(W_{\max }\left(A_{\otimes}^{m}\right)\right)}=\max _{1 \leq i \leq n} a_{i i} ;
$$


(ii) If $\max _{1 \leq i, j \leq n} a_{i j}=\max _{1 \leq i \leq n} a_{i i}$ and the limit $\lim _{m \rightarrow \infty} \frac{l\left(W_{\max }\left(A_{\otimes}^{m+1}\right)\right)}{l\left(W_{\max }\left(A_{\otimes}^{m}\right)\right)}$ exists, then it is equal to the maximum of $a_{i i}$ on $1 \leq i \leq n$.

Proof (i) By Propositions 3 and 4(v) we may assume without loss of generality that $a_{11} \leq a_{22} \leq \ldots \leq a_{n n}$ and that $A$ is upper triangular matrix. By computing $A_{\otimes}^{m}$, one can see that there exists some $s \geq n$ such that

$$
A_{\otimes}^{m}=\left[\begin{array}{cccccc}
a_{11}^{m} & k_{12} a_{22}^{m-1} & \cdots & k_{1 i} a_{i i}^{m-i+1} & \cdots & k_{1 n} a_{n n}^{m-n+1} \\
0 & a_{22}^{m} & \cdots & k_{2 i} a_{i i}^{m-i+2} & \cdots & k_{2 n} a_{n n}^{m-n+2} \\
\vdots & 0 & \ddots & \vdots & \cdots & \vdots \\
0 & \vdots & 0 & a_{i i}^{m} & \cdots & k_{i n} a_{n n}^{m-n+i} \\
\vdots & \vdots & \cdots & \ddots & \ddots & \vdots \\
0 & 0 & \cdots & \cdots & 0 & a_{n n}^{m}
\end{array}\right] \forall m \geq s
$$

We claim that there exist $1 \leq i_{0} \leq n$ and $s_{0} \geq n$ such that

$$
\max _{1 \leq i, j \leq n}\left(A_{\otimes}^{m}\right)_{i j}=k_{i_{0} n} a_{n n}^{m-n+i_{0}} \forall m \geq s_{0} .
$$

If this is not the case, then for some large enough $m \geq n$, there exist $1 \leq i_{1}, j_{1} \leq n$ such that

$$
k_{i_{1} j_{1}} a_{j_{1} j_{1}}^{m-j_{1}+i_{1}}>k_{i n} a_{n n}^{m-n+i}, 1 \leq i \leq n
$$

This shows that

$$
m<\frac{\ln \left(\frac{k_{i n} a_{j_{1} j_{1}}^{j_{1}-i_{1}}}{k_{i_{1} j_{1}} a_{n n}^{n-i}}\right)}{\ln \left(\frac{a_{j_{1} j_{1}}}{a_{n n}}\right)}
$$

which leads to a contradiction. This shows that the claim is true and the result follows since the minimal element on the diagonal of $A_{\otimes}^{m}$ is strictly smaller than $a_{n n}^{m}$ for all $m \geq s_{0}$.

(ii) We may assume that $a_{11} \leq a_{22} \leq \cdots \leq a_{n n}$. As $\max _{1 \leq i, j \leq n} a_{i j}=\max _{1 \leq i \leq n} a_{i i}=a_{n n}$, it follows that

$$
a_{n n}^{m}=\max _{1 \leq i=i_{0} \leq i_{1} \leq \cdots \leq i_{m-1} \leq j=i_{m} \leq n} a_{i i_{1}} a_{i_{1} i_{2}} \ldots a_{i_{m-1} j}, \quad \forall m \geq 1 .
$$

It now follows from (9) that

$$
\lim _{m \rightarrow \infty} \frac{l\left(W_{\max }\left(A_{\otimes}^{m+1}\right)\right)}{l\left(W_{\max }\left(A_{\otimes}^{m}\right)\right)}=\lim _{m \rightarrow \infty} \frac{a_{n n}^{m+1}-a_{11}^{m+1}}{a_{n n}^{m}-a_{11}^{m}}=a_{n n},
$$

which completes the proof. 


\section{Max joint numerical ranges}

Recall that the joint numerical range of a $k$-tuple $\left(A_{1}, \ldots, A_{k}\right)$, where $A_{i} \in M_{n}, i=1, \ldots, k$, is defined by $([2,12])$

$$
W\left(A_{1}, \ldots, A_{k}\right)=\left\{\left(x^{*} A_{1} x, \ldots, x^{*} A_{k} x\right): x \in S^{1}\right\},
$$

where $S^{1}=\left\{x \in \mathbb{C}^{n}: x^{*} x=1\right\}$. So, one can define the max joint numerical range of a $k$-tuple of $n \times n$ nonnegative matrices $\mathbb{A}=\left(A_{1}, \ldots, A_{k}\right)$ in the following way:

$$
W_{\max }(\mathbb{A})=\left\{\left(x^{t} \otimes A_{1} \otimes x, \ldots, x^{t} \otimes A_{k} \otimes x\right): x \in \mathbb{R}_{+}^{n}, x^{t} \otimes x=1\right\} .
$$

Remark 3 Note that it follows from the above definition that $\left(a_{1}, \ldots, a_{k}\right) \in W_{\max }\left(A_{1}, \ldots, A_{k}\right)$ if and only if there exists $x \in \mathbb{R}_{+}^{n}, x^{t} \otimes x=1$ such that $a_{i}=x^{t} \otimes A_{i} \otimes x$, for all $1 \leq i \leq k$.

From Theorem 2 and Remark 3 we conclude the following result.

Corollary 2 If $\mathbb{A}=\left(A_{1}, \ldots, A_{k}\right)$ such that $A_{l}=\left(a_{i j}^{(l)}\right) \in M_{n}\left(\mathbb{R}_{+}\right), l=1, \ldots, k$, then

$$
W_{\max }(\mathbb{A}) \subseteq\left[s_{1}, t_{1}\right] \times \cdots \times\left[s_{k}, t_{k}\right] \subseteq \mathbb{R}_{+}^{k},
$$

where $t_{l}=\max _{1 \leq i, j \leq n} a_{i j}^{(l)}$ and $s_{l}=\min _{1 \leq i \leq n} a_{i i}^{(l)}$ for all $1 \leq l \leq k$. Consequently, $W_{\max }(\mathbb{A})$ is $a$ compact set.

Next we define a max joint numerical range $W_{\max }(\Sigma)$ of a bounded set $\Sigma$ of $n \times n$ nonnegative matrices in the following way:

$$
W_{\max }(\Sigma)=\cup_{A \in \Sigma} W_{\max }(A) .
$$

By Theorem 2, we have

$$
W_{\max }(\Sigma)=\bigcup_{A \in \Sigma}\left[\min _{1 \leq i \leq n} a_{i i}, \max _{1 \leq i, j \leq n} a_{i j}\right] .
$$

Recall that the supremum matrix $S(\Sigma)$ is defined by

$$
S(\Sigma)_{i j}=\sup _{A \in \Sigma} a_{i j}
$$

and that the generalized (joint) spectral radius $\mu(\Sigma)$ of $\Sigma$ is equal to ([5, 6, 9, 10, 13])

$$
\mu(\Sigma)=\limsup _{m \rightarrow \infty}\left(\sup _{A \in \Sigma_{\otimes}^{m}} \mu(A)\right)^{\frac{1}{m}}=\sup _{m \in \mathbb{N}}\left(\sup _{A \in \Sigma_{\otimes}^{m}} \mu(A)\right)^{\frac{1}{m}}
$$


where $\Sigma_{\otimes}^{m}=\left\{A_{1} \otimes A_{2} \otimes \ldots \otimes A_{m}: A_{i} \in \Sigma\right\}$. The max Berger Wang formula asserts that $([9,10,13])$

$$
\mu(\Sigma)=\lim _{m \rightarrow \infty}\left(\sup _{A \in \Sigma_{\otimes}^{m}}\|A\|\right)^{\frac{1}{m}}=\inf _{m \in \mathbb{N}}\left(\sup _{A \in \Sigma_{\otimes}^{m}}\|A\|\right)^{\frac{1}{m}},
$$

where $\|A\|=\max _{i, j=1, \ldots, n} a_{i j}$ (since all norms on $\mathbb{R}^{n \times n}$ are equivalent one can use here any norm on $\left.\mathbb{R}^{n \times n}\right)$. We also have $\mu(\Sigma)=\mu(S(\Sigma)),\|\Sigma\| \underset{A \in \Sigma, i, j=1, \ldots, n}{=} \sup _{i j}=\|S(\Sigma)\|$ and

$$
\mu(\Sigma)=\limsup _{m \rightarrow \infty}\left(\sup _{A \in \Sigma_{\otimes}^{m}} t r_{\otimes}(A)\right)^{\frac{1}{m}}
$$

where $\operatorname{tr}(A)=\max _{i=1, \ldots, n} a_{i i}([10$, Theorem 3.3. $])$.

By (11), the following result follows.

Corollary 3 If $\Sigma$ is a bounded set of $n \times n$ nonnegative matrices, then

$$
\inf _{A \in \Sigma, i=1, \ldots, n} a_{i i}=\inf W_{\max }(\Sigma) \leq \mu(\Sigma) \leq \sup W_{\max }(\Sigma)=\|\Sigma\|
$$

\section{$4 k$-numerical range, $k$-geometric max spectrum and $k$-tropical spectrum}

Now, we introduce and study the max $k$-numerical range, where $k \leq n$ is a positive integer. Let $I_{k}$ denote the $k \times k$ identity matrix. A matrix $X \in M_{n \times k}\left(\mathbb{R}_{+}\right)$is called an isometry in max algebra if $X^{t} \otimes X=I_{k}$, and the set of all $n \times k$ isometry matrices in max algebra is denoted by $\mathcal{X}_{n \times k}$. For the case $k=n, \mathcal{X}_{n \times n}$ is equal to $\mathcal{U}_{n}$, which was introduced in Definition 2.

Definition 3 For $A \in M_{n}\left(\mathbb{R}_{+}\right)$with $k \leq n$, the max $k$-numerical range of $A$ is defined and denoted by

$$
\begin{aligned}
W_{\max }^{k}(A) & =\left\{\bigoplus_{i=1}^{k} x_{i}^{t} \otimes A \otimes x_{i}: X=\left[x_{1}, x_{2}, \ldots, x_{k}\right] \in \mathcal{X}_{n \times k}\right\} \\
& =\left\{t r_{\otimes}\left(X^{t} \otimes A \otimes X\right): X=\left[x_{1}, x_{2}, \ldots, x_{k}\right] \in \mathcal{X}_{n \times k}\right\} .
\end{aligned}
$$

It is clear that $W_{\max }^{1}(A)=W_{\max }(A)$, so the notion of max $k$-numerical range is a generalization of the max numerical range of matrices.

Note that

$$
t r_{\otimes}\left(X^{t} \otimes A \otimes X\right)=x_{1}^{t} \otimes A \otimes x_{1} \oplus x_{2}^{t} \otimes A \otimes x_{2} \oplus \cdots \oplus x_{k}^{t} \otimes A \otimes x_{k},
$$

where $1 \leq i, j \leq k$ and $x_{i}, x_{j} \in \mathbb{R}_{+}^{n}$, 


$$
x_{i}^{t} \otimes x_{j}=\delta_{i j}= \begin{cases}1 & i=j \\ 0 & i \neq j\end{cases}
$$

Remark 4 For a nonnegative matrix $A=\left(a_{i j}\right)$ and $1 \leq k \leq n$, the map $f_{A}: \mathcal{X}_{n \times k} \longrightarrow \mathbb{R}_{+}$is locally Lipschitz continuous on $\mathcal{X}_{n \times k}$, where

$$
\mathcal{X}_{n \times k}=\left\{X \in M_{n \times k}\left(\mathbb{R}_{+}\right): X^{t} \otimes X=I_{k}\right\}, f_{A}(X):=t r_{\otimes}\left(X^{t} \otimes A \otimes X\right)
$$

Note that $W_{\max }^{k}(A)$ is the image of the continuous function $f_{A}$. Using connectivity and compactness of $\mathcal{X}_{n \times k}, W_{\max }^{k}(A)$ is a connected and compact set.

We have the following explicit formula for $W_{\max }^{k}(A)$.

Theorem 3 Suppose that $A=\left(a_{i j}\right) \in M_{n}\left(\mathbb{R}_{+}\right)$and let $1 \leq k \leq n$ be a positive integer. We have

$$
W_{\max }^{k}(A)=[c, d]
$$

where $c=\min \left\{\bigoplus_{j=1}^{k} a_{i_{j} i_{j}}: 1 \leq i_{1}<i_{2}<\cdots<i_{k} \leq n\right\}$ and $d=\max _{1 \leq i, j \leq n} a_{i j}$.

Proof By Definition 3 and Theorem 2, it follows that $\min W_{\max }^{k}(A)=c$ and $\max W_{\max }^{k}(A)=d$. Since $W_{\max }$ is a connected set, by Remark 4, this implies (13).

In the following theorem, we state some basic properties of the max $k$-numerical range of matrices.

Theorem 4 Let $A \in M_{n}\left(\mathbb{R}_{+}\right)$and $1 \leq k \leq n$. Then the following assertions hold:

(i) $W_{\text {max }}^{k}(\alpha A \oplus \beta I)=\alpha W_{\text {max }}^{k}(A) \oplus \beta$ and $W_{\text {max }}^{k}(A \oplus B) \subseteq W_{\text {max }}^{k}(A) \oplus W_{\text {max }}^{k}(B)$, where $\alpha, \beta \in \mathbb{R}_{+}$and $B \in M_{n}\left(\mathbb{R}_{+}\right)$;

(ii) $W_{\max }^{k}\left(U^{t} \otimes A \otimes U\right)=W_{\max }^{k}(A)$ if $U \in \mathcal{U}_{n}$;

(iii)f $B \in M_{m}\left(\mathbb{R}_{+}\right)$is a principal submatrix of $A$ and $k \leq m$, then $W_{\text {max }}^{k}(B) \subseteq W_{\max }^{k}(A)$.

Consequently, if $V=\left[e_{i_{1}}, e_{i_{2}}, \ldots, e_{i_{s}}\right] \in M_{n \times s}\left(\mathbb{R}_{+}\right)$, where $1 \leq s \leq n$, then

$W_{\max }^{k}\left(V^{t} \otimes A \otimes V\right) \subseteq W_{\max }^{k}(A)$, and the equality holds if $\mathrm{s}=\mathrm{n}$;

(iv) $W_{\max }^{k}\left(A^{t}\right)=W_{\max }^{k}(A)$;

(v)If $k<n$, then $W_{\max }^{k+1}(A) \subseteq W_{\max }^{k}(A)$. Consequently,

$$
W_{\max }^{n}(A) \subseteq W_{\max }^{n-1}(A) \subseteq \cdots \subseteq W_{\max }^{2}(A) \subseteq W_{\max }(A) .
$$

Proof The properties (i), (ii), (iii) and (iv) easily follow from Theorem 3 (or directly from Definition 3). 
To prove $(v)$, let $z \in W_{\max }^{k+1}(A)$ be given. So, by Definition 3 , there exist $1 \leq i_{1} \leq i_{2} \leq \ldots \leq i_{k} \leq i_{k+1} \leq n, X=\left[x_{i_{1}}, x_{i_{2}}, \ldots, x_{i_{k}}, x_{i_{k+1}}\right] \in \mathcal{X}_{n \times(k+1)}$ such that

$$
z=\bigoplus_{i=i_{1}}^{i_{k+1}} x_{i}^{t} \otimes A \otimes x_{i}
$$

Now, one can assume that, without loss of generality,

$$
x_{i_{1}}^{t} \otimes A \otimes x_{i_{1}} \leq \cdots \leq x_{i_{k}}^{t} \otimes A \otimes x_{i_{k}} \leq x_{i_{k+1}}^{t} \otimes A \otimes x_{i_{k+1}} .
$$

Hence, by setting $X=\left[x_{i_{2}}, \ldots, x_{i_{k}}, x_{i_{k+1}}\right]$, we have $X \in \mathcal{X}_{n \times k}$ and so

$$
z=\bigoplus_{i=i_{2}}^{i_{k+1}} x_{i}^{t} \otimes A \otimes x_{i}
$$

This implies that $z \in W_{\max }^{k}(A)$ and the proof is complete.

The following example illustrates Theorem $4(\mathrm{v})$.

\section{Example 3 If}

$$
A=\left[\begin{array}{llll}
4 & 7 & 5 & 8 \\
8 & 2 & 0 & 7 \\
2 & 8 & 1 & 4 \\
1 & 6 & 2 & 2
\end{array}\right],
$$

then

$$
W_{\max }^{1}(A)=W_{\max }(A)=[1,8], W_{\max }^{2}(A)=[2,8], W_{\max }^{3}(A)=[2,8], W_{\max }^{4}(A)=[4,8] .
$$

Similarly as in the classical linear case, we define below max $k$-geometric spectrum and $k$-tropical spectrum of $A \in M_{n}\left(\mathbb{R}_{+}\right)$.

Definition 4 Let $A \in M_{n}\left(\mathbb{R}_{+}\right), 1 \leq k \leq n$ and let $\mu_{1}, \ldots, \mu_{n} \in \sigma_{\text {max }}(A)$ counting standard vector multiplicities. The max $k$-geometric spectrum of $A$ is defined by

$$
\sigma_{\max }^{k}(A)=\left\{\bigoplus_{j=1}^{k} \mu_{i_{j}}: 1 \leq i_{1}<i_{2}<\cdots<i_{k} \leq n\right\} .
$$

Definition 5 Let $A \in M_{n}\left(\mathbb{R}_{+}\right), 1 \leq k \leq n$ and let $\lambda_{1}, \ldots, \lambda_{n} \in \sigma_{\text {trop }}(A)$ counting tropical multiplicities. The $k$-tropical max spectrum of $A$ is defined by

$$
\sigma_{\text {trop }}^{k}(A)=\left\{\bigoplus_{j=1}^{k} \lambda_{i_{j}}: 1 \leq i_{1}<i_{2}<\cdots<i_{k} \leq n\right\} .
$$

It is clear that $\sigma_{\max }^{1}(A)=\sigma_{\max }(A)$ and $\sigma_{\text {trop }}^{1}(A)=\sigma_{\text {trop }}(A)$. 
Recall that the max convex hull of a set $M \subseteq \mathbb{R}_{+}$, which is denoted by $\operatorname{conv}_{\otimes}(M)$, is defined as the set of all max convex linear combinations of elements of $M$, i.e.,

$$
\operatorname{conv}_{\otimes}(M):=\left\{\bigoplus_{i=1}^{m} \alpha_{i} x_{i}: m \in \mathbb{N}, x_{i} \in M, \alpha_{i} \geq 0, i=1, \ldots, m, \bigoplus_{i=1}^{m} \alpha_{i}=1\right\}
$$

By Definitions 4 and 5, it is obvious that

$$
\begin{aligned}
& \operatorname{conv}_{\otimes}\left(\sigma_{\max }^{k}(A)\right)=\left[\begin{array}{c}
\min _{1 \leq i_{1}<i_{2}<\cdots<i_{k} \leq n} \mu_{i_{j}}, \max _{1 \leq n} \\
\mu_{i}
\end{array}\right], \forall 1 \leq k \leq n, 1 \leq j \leq k, \\
& \operatorname{conv}_{\otimes}\left(\sigma_{\text {trop }}^{k}(A)\right)=\left[\begin{array}{c}
\min \bigoplus_{1 \leq i_{1}<i_{2}<\cdots<i_{k} \leq n} \lambda_{i_{i}}, \max _{1 \leq i \leq n} \lambda_{i}
\end{array}\right], \forall 1 \leq k \leq n, 1 \leq j \leq k .
\end{aligned}
$$

Since $\sigma_{\max }(A) \subset \sigma_{\text {trop }}(A) \subset W_{\text {max }}(A)$, the following result follows from Definitions 4 , 5 and 3 and Theorems 2 and 3 .

Proposition 7 Let $A \in M_{n}\left(\mathbb{R}_{+}\right)$and $1 \leq k \leq n$. Then $\operatorname{conv}_{\otimes}\left(\sigma_{\max }^{k}(A)\right) \subset W_{\max }^{k}(A)$ and $\operatorname{conv}_{\otimes}\left(\sigma_{\text {trop }}^{k}(A)\right) \subset W_{\max }^{k}(A)$.

By Theorem 3, (14) and (15), we have the following two results.

Proposition 8 Let $A \in M_{n}\left(\mathbb{R}_{+}\right), 1 \leq k \leq n$ and let $\mu_{1}, \ldots, \mu_{n} \in \sigma_{\max }(A)$ counting standard vector multiplicities. If $\min _{1 \leq i_{1}<\cdots<i_{k} \leq n} \oplus \mu_{i_{j}}=\min _{1 \leq i_{1}<\cdots<i_{k} \leq n} \oplus a_{i_{j} i_{j}}$ and $\mu(A)=\max _{1 \leq i, j \leq n} a_{i j}$, then

$$
\operatorname{conv}_{\otimes}\left(\sigma_{\max }^{k}(A)\right)=W_{\max }^{k}(A) .
$$

Proposition 9 Let $A \in M_{n}\left(\mathbb{R}_{+}\right), 1 \leq k \leq n$ and let $\lambda_{1}, \ldots, \lambda_{n} \in \sigma_{\text {trop }}(A)$ counting tropical multiplicities. If $\min _{1 \leq i_{1}<\cdots<i_{k} \leq n} \oplus \lambda_{i_{j}}=\min _{1 \leq i_{1}<\cdots<i_{k} \leq n} \oplus a_{i_{j} i_{j}}$ and $\mu(A)=\max _{1 \leq i, j \leq n} a_{i j}$, then

$$
\operatorname{conv}_{\otimes}\left(\sigma_{\text {trop }}^{k}(A)\right)=W_{\max }^{k}(A) \text {. }
$$

Remark 5 Let $A \in M_{n}\left(\mathbb{R}_{+}\right)$be irreducible and let $1 \leq k \leq n$. Then $\mu(A)$ is a unique geometric max eigenvalue of $A$, and so for all $1 \leq k \leq n$, we have

$$
\sigma_{\max }^{k}(A)=W_{\max }^{k}(B)=\{\mu(A)\},
$$

where $B=\left(b_{i j}\right) \in M_{n}\left(\mathbb{R}_{+}\right)$such that $b_{i i}=\mu(A)=\max _{1 \leq i, j \leq n} b_{i j}, 1 \leq i \leq n$.

In the following result we state the relationship between the max $k$-geometric spectrums of $A$.

Proposition 10 Let $A \in M_{n}\left(\mathbb{R}_{+}\right)$and $1 \leq k<n$. Then $\sigma_{\max }^{k+1}(A) \subseteq \sigma_{\max }^{k}(A)$. Consequently, 


$$
\{\mu(A)\}=\sigma_{\max }^{n}(A) \subseteq \sigma_{\max }^{n-1}(A) \subseteq \cdots \subseteq \sigma_{\max }^{2}(A) \subseteq \sigma_{\otimes}(A) .
$$

Proof At first, let $z \in \sigma_{\max }^{k+1}(A)$ be given. By Definition 4 there exists $1 \leq i_{1}<i_{2}<\cdots<i_{k+1} \leq n \quad$ such $\quad$ that $\quad z=\bigoplus_{j=1} \mu_{i_{j}} . \quad$ Since $z=\bigoplus_{j=1}^{k+1} \mu_{i_{j}}=\bigoplus_{p=1}^{k} \mu_{j_{p}} \in \sigma_{\max }^{k}(A)$, the proof is complete.

The following example from [11] illustrates the result above.

\section{Example 4 Let}

$$
A=\left[\begin{array}{lllll}
2 & 0 & 0 & 0 & 0 \\
1 & 3 & 0 & 0 & 0 \\
1 & 0 & 1 & 1 & 0 \\
0 & 0 & 1 & 2 & 0 \\
0 & 0 & 0 & 0 & 1
\end{array}\right] .
$$

Then [11, Example 2.13.],

$$
r_{e_{1}}(A)=r_{e_{2}}(A)=3, r_{e_{3}}(A)=r_{e_{4}}(A)=2, r_{e_{5}}(A)=1,
$$

where the $r_{e_{j}}(A)$ 's are the max geometric eigenvalues of $A$. One can easily check that

$$
\sigma_{\max }^{5}(A)=\{3\}, \sigma_{\max }^{4}(A)=\{3\}, \sigma_{\max }^{3}(A)=\{2,3\}, \sigma_{\max }^{2}(A)=\{2,3\}, \sigma_{\max }^{1}(A)=\{1,2,3\} .
$$

Also

$$
\operatorname{conv}_{\otimes}\left(\sigma_{\max }^{1}(A)\right)=[1,3], \operatorname{conv}_{\otimes}\left(\sigma_{\max }^{2}(A)\right)=[2,3], \operatorname{conv}_{\otimes}\left(\sigma_{\max }^{3}(A)\right)=[2,3],
$$

$\operatorname{conv}_{\otimes}\left(\sigma_{\max }^{4}(A)\right)=\{3\}$ and $\operatorname{conv} v_{\otimes}\left(\sigma_{\max }^{5}(A)\right)=\{3\}$.

The following analogue of Proposition 10 for the $k$-tropical spectrum is proved in a similar way as Proposition 10.

Proposition 11 Let $A \in M_{n}\left(\mathbb{R}_{+}\right)$and $1 \leq k<n$. Then $\sigma_{\text {trop }}^{k+1}(A) \subseteq \sigma_{\text {trop }}^{k}(A)$. Consequently,

$$
\{\mu(A)\}=\sigma_{\text {trop }}^{n}(A) \subseteq \sigma_{\text {trop }}^{n-1}(A) \subseteq \cdots \subseteq \sigma_{\text {trop }}^{2}(A) \subseteq \sigma_{\text {trop }}(A) .
$$

Remark 6 It is also possible to consider the max algebra analogues of a related object from the usual algebra, namely the max algebra analogues of a higher-rank numerical range of a matrix $B$ (cf. $[4,16])$. We do not study these objects in this article and leave this for further research. 


\section{Max c-numerical range and max C-numerical range}

Let $A, C \in M_{n}\left(\mathbb{R}_{+}\right)$and $c \in \mathbb{R}_{+}^{n}$. Next we define and study max $c$-numerical range and max $C$-numerical range of $A \in M_{n}\left(\mathbb{R}_{+}\right)$. To access more information about some known results in the complex case, see [8, Chapter1].

Definition 6 Let $A \in M_{n}\left(\mathbb{R}_{+}\right)$and $c=\left[c_{1}, c_{2}, \ldots, c_{n}\right]^{t} \in \mathbb{R}_{+}^{n}$. The max $c$-numerical range of $A$ is defined and denoted by

$$
W_{\text {max }}^{c}(A)=\left\{\bigoplus_{i=1}^{n} c_{i} x_{i}^{t} \otimes A \otimes x_{i}: X=\left[x_{1}, x_{2}, \ldots, x_{n}\right] \in M_{n}\left(\mathbb{R}_{+}\right), X^{t} \otimes X=I_{n}\right\} .
$$

In Definition 6, it's obvious that $X \in \mathcal{U}_{n}$, where the notation $\mathcal{U}_{n}$ was denoted in (2).

It is clear that $W_{\max }^{c}(A)=\left\{\operatorname{tr}_{\otimes}\left(C \otimes X^{t} \otimes A \otimes X\right): X \in \mathcal{U}_{n}\right\}$, where $C=\operatorname{diag}\left(c_{1}, \ldots, c_{n}\right), c=\left[c_{1}, c_{2}, \ldots, c_{n}\right]^{t} \in \mathbb{R}_{+}^{n}$. Also, one can easily verify

$$
W_{\max }^{c}(A)=\left\{c_{k}\left(\oplus_{i=1}^{n} a_{i i}\right): k=1,2, \ldots, n\right\},
$$

and, consequently,

$$
\operatorname{conv}_{\otimes}\left(W_{\max }^{c}(A)\right)=\left[\min _{1 \leq k \leq n} c_{k}\left(\oplus_{i=1}^{n} a_{i i}\right), \bigoplus_{k=1}^{n} c_{k}\left(\oplus_{i=1}^{n} a_{i i}\right)\right] .
$$

Remark 7 Let $A \in M_{n}\left(\mathbb{R}_{+}\right)$and $c=[\alpha, \ldots, \alpha]^{t} \in \mathbb{R}_{+}^{n}$. Then

$$
W_{\max }^{c}(A)=\left\{t r_{\otimes}\left(\alpha X^{t} \otimes A \otimes X\right): X \in \mathcal{U}_{n}\right\} .
$$

Since $t r_{\otimes}\left(X^{t} \otimes A \otimes X\right)=t r_{\otimes}(A)$ for $X \in \mathcal{U}_{n}$ it follows that

$$
W_{\max }^{c}(A)=\left\{\alpha t r_{\otimes}(A)\right\} .
$$

Next we introduce and study the notion of max $C$-numerical range of nonnegative matrices, where $C \in M_{n}\left(\mathbb{R}_{+}\right)$.

Definition 7 Let $A, C \in M_{n}\left(\mathbb{R}_{+}\right)$. The max $C$-numerical range of $A$ is defined and denoted by

$$
W_{\max }^{C}(A)=\left\{t r_{\otimes}\left(C \otimes X^{t} \otimes A \otimes X\right): X=\left[x_{1}, x_{2}, \ldots, x_{n}\right] \in \mathcal{U}_{n}\right\}
$$

Example 5 Let $C=\left(c_{i j}\right) \in M_{n}\left(\mathbb{R}_{+}\right)$such that $c_{11}=1$ and $c_{i j}=0$ elsewhere. Then one can easily obtain that

$$
\operatorname{conv}_{\otimes}\left(W_{\max }^{C}(A)\right)=\left[\min _{1 \leq i \leq n} a_{i i}, \bigoplus_{i=1}^{n} a_{i i}\right]
$$

In the following theorem, we state some basic properties of the max $C$-numerical range of non-negative matrices. 
Theorem 5 Let $A, C \in M_{n}\left(\mathbb{R}_{+}\right)$. Then the following assertions hold:

(i) $W_{\max }^{C}\left(\alpha A \oplus \beta I_{n}\right)=\alpha W_{\max }^{C}(A) \oplus \beta \operatorname{tr}_{\otimes}(C)$, where $\alpha, \beta \in \mathbb{R}_{+}$;

(ii) $W_{\max }^{C}(A \oplus B) \subseteq W_{\max }^{C}(A) \oplus W_{\max }^{C}(B)$ and $W_{\max }^{C \oplus D}(A) \subseteq W_{\max }^{C}(A) \oplus W_{\max }^{D}(A)$, where $B, D \in M_{n}\left(\mathbb{R}_{+}\right)$;

(iii) $W_{\max }^{C}\left(U^{t} \otimes A \otimes U\right)=W_{\max }^{C}(A)$, where $U \in U_{n}$;

(iv) If $C^{t}=C$, then $W_{\max }^{C}\left(A^{t}\right)=W_{\max }^{C}(A)$;

(v) If $C=\alpha I_{n}$, where $\alpha \in \mathbb{R}_{+}$, then $W_{\max }^{C}(A)=\left\{\alpha \operatorname{tr}_{\otimes}(A)\right\}$.

(vi) $W_{\max }^{C}(A)=W_{\text {max }}^{A}(C)$.

(vii) $W_{\max }^{V \otimes C} \otimes V(A)=W_{\max }^{C}(A)$, where $V \in \mathcal{U}_{n}$.

Proof The assertions (i), (ii), (iii), (iv), (v) and (vi) follow easily from Definition 7.

To prove (vii), let $z \in W_{\max }^{V^{\prime} \otimes C \otimes V}(A)$ be given. Then by Definition 7, there exists $X \in \mathcal{U}_{n}$, such that $z=\operatorname{tr}_{\otimes}\left(V^{t} \otimes C \otimes V \otimes X^{t} \otimes A \otimes X\right)$. Since

$$
t r_{\otimes}\left(V^{t} \otimes C \otimes V \otimes X^{t} \otimes A \otimes X\right)=t r_{\otimes}\left(C \otimes\left(X \otimes V^{t}\right)^{t} \otimes A \otimes\left(X \otimes V^{t}\right)\right) .
$$

By setting $U=X \otimes V^{t} \in \mathcal{U}_{n}$, one has $z \in W_{\max }^{C}(A)$, and so

$$
W_{\max }^{V^{+} \otimes C \otimes V}(A) \subseteq W_{\max }^{C}(A) .
$$

In a similar way the reverse inclusion can easily be verified.

The following result is a special case of Theorem5.

Corollary 4 Let $A \in M_{n}\left(\mathbb{R}_{+}\right)$and $c=\left[c_{1}, \ldots, c_{n}\right]^{t} \in \mathbb{R}_{+}^{n}$. Then the following assertions hold:

(i) $W_{\text {max }}^{c}\left(\alpha A \oplus \beta I_{n}\right)=\alpha W_{\text {max }}^{c}(A) \oplus \beta \bigoplus_{i=1}^{n} c_{i}$, where $\alpha, \beta \in \mathbb{R}_{+}$;

(ii) $W_{\max }^{c}\left(U^{t} \otimes A \otimes U\right)=W_{\max }^{c}(A)$, where $U \in \mathcal{U}_{n}$;

(iii) $W_{\max }^{c}(A \oplus B) \subseteq W_{\max }^{c}(A) \oplus W_{\max }^{c}(B)$ and $W_{\max }^{c \oplus d}(A) \subseteq W_{\max }^{c}(A) \oplus W_{\max }^{d}(A)$, where $d \in \mathbb{R}_{+}^{n}$ and $B \in M_{n}\left(\mathbb{R}_{+}\right)$

(iv) $W_{\max }^{c}\left(A^{t}\right)=W_{\max }^{c}(A)$;

(v)If $A=\alpha I_{n}$, where $\alpha \in \mathbb{R}_{+}$, then $W_{\max }^{c}(A)=\left\{\alpha \bigoplus_{i=1}^{n} c_{i}\right\}$.

Acknowledgements The third author was supported in part by the Slovenian Research Agency (grants P1-0222, J1-8133, J1-8155, and N1-0071). We thank the referees for very useful comments and corrections.

Open Access This article is licensed under a Creative Commons Attribution 4.0 International License, which permits use, sharing, adaptation, distribution and reproduction in any medium or format, as long as you give appropriate credit to the original author(s) and the source, provide a link to the Creative Commons licence, and indicate if changes were made. The images or other third party material in this article 
are included in the article's Creative Commons licence, unless indicated otherwise in a credit line to the material. If material is not included in the article's Creative Commons licence and your intended use is not permitted by statutory regulation or exceeds the permitted use, you will need to obtain permission directly from the copyright holder. To view a copy of this licence, visit http://creativecommons.org/licen ses/by/4.0/.

\section{References}

1. Aghamollaei, G.H., Ghasemizadeh, N.: Some results on matrix polynomials in the max algebra. Banach J. Math. Anal. 9, 17-26 (2015)

2. Binding, P., Li, C.K.: Joint ranges of Hermitian matrices and simultaneous diagonalization. Linear Algebra Appl. 151, 157-167 (1991)

3. Butkovič, P.: Max-linear Systems: Theory and Algorithms. Springer-Verlag, London (2010)

4. Gau, H.L., Wu, P.Y.: Higher-rank numerical ranges and Kippenhahn polynomials. Linear Algebra Appl. 438, 3054-3061 (2013)

5. Guglielmi, N., Mason, O., Wirth, F.: Barabanov norms, Lipschitz continuity and monotonicity for the max algebraic joint spectral radius. Linear Algebra Appl. 550, 37-58 (2018)

6. Gursoy, B.B., Mason, O.: Spectral properties of matrix polynomials in the max algebra. Linear Algebra Appl. 435, 1626-1630 (2011)

7. Gutkin, E., Jonckheere, E.A., Karow, M.: Convexity of the joint numerical range: topological and differential geometric viewpoints. Linear Algebra Appl. 376, 143-171 (2004)

8. Horn, R.A., Johnson, C.R.: Topics in Matrix Analysis. Cambridge University Press, Cambridge (1991)

9. Lur, Y.Y.: A max version of the generalized spectral radius theorem. Linear Algebra Appl. 418, 336-346 (2006)

10. Müller, V., Peperko, A.: Generalized spectral radius and its max algebra version. Linear Algebra Appl. 439, 1006-1016 (2013)

11. Müller, V., Peperko, A.: On the spectrum in max algebra. Linear Algebra Appl. 485, 250-266 (2015)

12. Müller, V., Tomilov, Y.: Joint numerical ranges and compressions of powers of operators. J. Lond. Math. Soc. 99, 127-152 (2019)

13. Peperko, A.: On the max version of the generalized spectral radius theorem. Linear Algebra Appl. 428, 2312-2318 (2008)

14. Rosenmann, A., Lehner, F., Peperko, A.: Polynomial convolutions in max-plus algebra. Linear Algebra Appl. 578, 370-401 (2019)

15. Tavakolipour, H., Shakeri, F.: On the numerical range in tropical algebra. Linear Multilinear Algebra 67, (2019). https://doi.org/10.1080/03081087.2018.1478946

16. Woerderman, H.: The higher rank numerical range is convex. Linear Multilinear Algebra 56, 65-67 (2008) 